\title{
ON THE DISTRIBUTION OF COMPLEX ROOTS OF RANDOM POLYNOMIALS WITH HEAVY-TAILED COEFFICIENTS
}

\author{
F. GÖTZE, D. ZAPOROZHETS
}

\begin{abstract}
Consider a random polynomial $G_{n}(z)=\xi_{n} z^{n}+\cdots+\xi_{1} z+\xi_{0}$ with i.i.d. complex-valued coefficients. Suppose that the distribution of $\log (1+$ $\left.\log \left(1+\left|\xi_{0}\right|\right)\right)$ has a slowly varying tail. Then the distribution of the complex roots of $G_{n}$ concentrates in probability, as $n \rightarrow \infty$, to two centered circles and is uniform in the argument as $n \rightarrow \infty$. The radii of the circles are $\left|\xi_{0} / \xi_{\tau}\right|^{1 / \tau}$ and $\left|\xi_{\tau} / \xi_{n}\right|^{1 /(n-\tau)}$, where $\xi_{\tau}$ denotes the coefficient with the maximum modulus.

Key words and concepts: roots of a random polynomial, roots concentration, heavy-tailed coefficients
\end{abstract}

\section{INTRODUCTION}

Consider the sequence of random polynomials

$$
G_{n}(z)=\xi_{n} z^{n}+\xi_{n-1} z^{n-1}+\cdots+\xi_{1} z+\xi_{0}
$$

where $\xi_{0}, \xi_{1}, \ldots, \xi_{n}, \ldots$ are i.i.d. real- or complex-valued random variables. We would like to investigate the behaviour of the complex roots of $G_{n}$.

The first results in this questions are due to Hammersley [2]. He derived an explicit formula for the $r$-point correlation function $(1 \leqslant r \leqslant n)$ of the roots of $G_{n}$ when the coefficients have an arbitrary joint distribution.

Shparo and Shur [9] showed that under quite general assumptions the roots of $G_{n}$ concentrate near the unit circle as $n$ tends to $\infty$ with asymptotically uniform distribution of the argument. More precisely, denote by $R_{n}(a, b)$ respectively $S_{n}(\alpha, \beta)$ the number of the roots of $G_{n}$ contained in the ring $\{z \in \mathbb{C}: a \leq|z| \leq b\}$ respectively the sector $\{z \in \mathbb{C}: \alpha \leq \arg z \leq \beta\}$. For $\varepsilon>0, m \in \mathbb{Z}_{+}$consider the function

$$
f(t)=[\underbrace{\log ^{+} \log ^{+} \ldots \log ^{+} t}_{m+1}]^{1+\varepsilon} \cdot \prod_{k=1}^{m} \underbrace{\log ^{+} \log ^{+} \ldots \log ^{+} t}_{k},
$$

where $\log ^{+} s=\max (1, \log s)$. If for some $\varepsilon>0, m \in \mathbb{Z}^{+}$

$$
\mathbf{E} f\left(\left|\xi_{0}\right|\right)<\infty
$$

then for any $\delta \in(0,1)$ and any $\alpha, \beta$ such that $-\pi \leqslant \alpha<\beta \leqslant \pi$

$$
\begin{array}{ll}
\frac{1}{n} R_{n}(1-\delta, 1+\delta) \stackrel{\mathbf{P}}{\longrightarrow} 1, & n \rightarrow \infty, \\
\frac{1}{n} S_{n}(\alpha, \beta) \stackrel{\mathbf{P}}{\longrightarrow} \frac{\beta-\alpha}{2 \pi}, \quad n \rightarrow \infty .
\end{array}
$$

Partially supported by RFBR (10-01-00242), RFBR-DFG (09-0191331), NSh-4472.2010.1, and CRC 701 "Spectral Structures and Topological Methods in Mathematics". 
Ibragimov and Zaporozhets 4] improved this result as follows. They showed that

$$
\mathbf{P}\left\{\frac{1}{n} R_{n}(1-\delta, 1+\delta) \underset{n \rightarrow \infty}{\longrightarrow} 1\right\}=1
$$

holds for any $\delta \in(0,1)$ if and only if

$$
\mathbf{E} \log \left(1+\left|\xi_{0}\right|\right)<\infty
$$

They also proved that for any $\alpha, \beta$ such that $-\pi \leqslant \alpha<\beta \leqslant \pi$

$$
\mathbf{P}\left\{\frac{1}{n} S_{n}(\alpha, \beta) \underset{n \rightarrow \infty}{\longrightarrow} \frac{\beta-\alpha}{2 \pi}\right\}=1
$$

holds for any distribution of $\xi_{0}$.

Shepp and Vanderbei [8] considered real-valued standard Gaussian coefficients and proved that

$$
\frac{1}{n} \mathbf{E} R_{n}\left(e^{-\delta / n}, e^{\delta / n}\right) \longrightarrow \frac{1+e^{-2 \delta}}{1-e^{-2 \delta}}-\frac{1}{\delta}, \quad n \rightarrow \infty
$$

for any $\delta>0$. Ibragimov and Zeitouni [3] extended this relation to the case of arbitrary i.i.d. coefficients from the domain of attraction of an $\alpha$-stable law:

$$
\frac{1}{n} \mathbf{E} R_{n}\left(e^{-\delta / n}, e^{\delta / n}\right) \longrightarrow \frac{1+e^{-\alpha \delta}}{1-e^{-\alpha \delta}}-\frac{2}{\alpha \delta}, \quad n \rightarrow \infty .
$$

It is interesting to consider the limit case when $\alpha \rightarrow 0$. Then

$$
\frac{1+e^{-\alpha \delta}}{1-e^{-\alpha \delta}}-\frac{2}{\alpha \delta} \longrightarrow 0
$$

and a natural assumption for the coefficient distribution would be a slowly varying tail. In this case (1) becomes

$$
\frac{1}{n} \mathbf{E} R_{n}\left(e^{-\delta / n}, e^{\delta / n}\right) \longrightarrow 0, \quad n \rightarrow \infty .
$$

This result (in a slightly stronger form) is proved in Theorem 1

In contrast to the concentration near the unit circumference, there exist random polynomials with quite a different asymptotic behavior of complex roots. Zaporozhets 10] constructed a random polynomial with i.i.d. coefficients such that in average $n / 2+o(1)$ of the complex roots concentrate near the origin and the same number tends to infinity as $n \rightarrow \infty$ (moreover, the expected number of real roots of this polynomial is at most 9 for all $n$ ). Theorem 2 generalizes this result.

The paper is organized as follows. In Sect. 2 we formulate our results. In Sect. 3 we prove some auxiliary lemmas. The theorems are proved in Sect. 4.

By $\sum_{j}$ we always denote a summation taken over all $j$ from $\{0,1, \ldots, n\}$. If conditions are stated for the summation, they are applied to this default range $j$ from $\{0,1, \ldots, n\}$.

\section{Results}

For the sake of simplicity, we assume that $\mathbf{P}\left\{\xi_{0}=0\right\}=0$. To treat the general case it is enough to study in the same way the behavior of the roots on the sets $\left\{\alpha_{n}=k, \beta_{n}=l\right\}$, where

$$
\alpha_{n}=\max \left\{j=0, \ldots, n: \xi_{j} \neq 0\right\}, \quad \beta_{n}=\min \left\{j=0, \ldots, n: \xi_{j} \neq 0\right\} .
$$


Theorem 1. If the distribution of $\left|\xi_{0}\right|$ has a slowly varying tail, then for any $\delta>0$

$$
\mathbf{P}\left\{R_{n}\left(e^{-\delta / n}, e^{\delta / n}\right)=0\right\} \longrightarrow 1, \quad n \rightarrow \infty .
$$

Consider the index $\tau=\tau_{n} \in\{0, \ldots, n\}$ such that $\left|\xi_{\tau}\right| \geqslant\left|\xi_{j}\right|$ for $j=0, \ldots, n$. If it is not unique, we take the minimum one. Let $\omega_{1}, \ldots, \omega_{n}$ be the complex roots of the system of equations

$$
z^{\tau}+\frac{\xi_{0}}{\xi_{\tau}}=0, \quad z^{n-\tau}+\frac{\xi_{\tau}}{\xi_{n}}=0 .
$$

Theorem 2. If the distribution of $\log \left(1+\log \left(1+\left|\xi_{0}\right|\right)\right)$ has a slowly varying tail, then for any $\varepsilon \in(0,1)$

$$
\mathbf{P}\left\{F_{n}(\varepsilon)\right\} \rightarrow 1, \quad n \rightarrow \infty,
$$

where $F_{n}(\varepsilon)$ denotes the event that it is possible to enumerate the roots $z_{1}, \ldots, z_{n}$ of $G_{n}$ in such a way that

$$
\left|z_{k}-w_{k}\right|<\frac{\varepsilon}{n}\left|w_{k}\right|
$$

for $k=1, \ldots, n$.

\section{Auxiliary lemmas}

First we need to formulate and prove some auxiliary results. The following result is due to Pellet.

Lemma 1. Let $g(z)=\sum_{j} a_{j} z^{j}$ be a polynomial of degree $n$. Suppose for some $k=1, \ldots, n-1$ the associated polynomial

$$
\tilde{g}(z)=\sum_{j \neq k}\left|a_{j}\right| z^{j}-\left|a_{k}\right| z^{k}
$$

has exactly two positive roots $R$ and $r, R>r$. Then $g$ has exactly $k$ roots inside the circle $\{z \in \mathbb{C}:|z|=r\}$ and $n-k$ roots outside the circle $\{z \in \mathbb{C}:|z|=R\}$.

Proof. See, e.g., [7].

The next lemma is due to Ostrowski.

Lemma 2. Let $B$ be a closed region in the complex plane, the boundary of which consists of a finite number of regular arcs; let the functions $f(z), h(z)$ be regular on $B$. Assume that for all values of the real parameter $t$, running in the interval $a \leqslant t \leqslant b$, the function $f(z)+t \cdot h(z)$ is non zero on the boundary of $B$. Then the number of the roots of $f(z)+t \cdot h(z)$ inside $B$ is independent of $t$ for $a \leqslant t \leqslant b$.

Proof. See $[$.

Lemma 3. Consider a monic polynomial of degree $n$ with complex coefficient $g(z)=\sum_{j} a_{j} z^{j}$ such that $a_{n}=1, a_{0} \neq 0$. Fix some $k=1, \ldots, n-1$ and denote by $w_{1}, \ldots, w_{n-k}$ the roots of the equation $z^{n-k}+a_{k}=0$. Put

$$
A_{k}=\sum_{j \neq k}\left|a_{j}\right|
$$

If for some $\varepsilon>0$

$$
A_{k} \leqslant\left(1-\frac{\varepsilon}{n}\right)\left(\frac{\varepsilon}{n+\varepsilon}\right)^{n-k}\left|a_{k}\right|^{1 /(n-k)}
$$


then $g$ has exactly $n-k$ roots $z_{1}, \ldots, z_{n-k}$ outside the unit circumference and it is possible to enumerate these roots in such a way that

$$
\left|z_{j}-w_{j}\right| \leqslant \frac{\varepsilon}{n}\left|w_{j}\right|
$$

for $j=1, \ldots, n-k$.

Proof. We will prove a stronger version of the Lemma 3 . Namely, we will show that the statement holds for the family of polynomials

$$
g_{t}(z)=z^{n}+a_{k} z^{k}+t \sum_{j \neq k, n} a_{j} z^{j}, \quad 0 \leqslant t \leqslant 1 .
$$

In particular,

$$
g_{0}(z)=z^{n}+a_{k} z^{k}, \quad g_{1}(z)=g(z) .
$$

Let us use Lemma 1 to estimate absolute values of the roots of $g_{t}$. Consider the associated polynomial

$$
\tilde{g}_{t}(z)=z^{n}-\left|a_{k}\right| z^{k}+t \sum_{j \neq k, n}\left|a_{j}\right| z^{j} .
$$

We have $\tilde{g}_{t}(0), \tilde{g}_{t}(\infty)>0$ and it follows from (2) that $\tilde{g}_{t}(1)<1$. Also, by Descarte's rule of signs, $\tilde{g}_{t}$ has at most 2 positive roots. Therefore $\tilde{g}_{t}$ has exactly 2 positive roots $r_{t}$ and $R_{t}$ such that

$$
0<r_{t}<1<R_{t}
$$

Now let us show that

$$
\left(1-\frac{\varepsilon}{n}\right)\left|a_{k}\right|^{1 /(n-k)} \leqslant R_{t} \leqslant\left|a_{k}\right|^{1 /(n-k)} .
$$

Since $\tilde{g}_{t}\left(R_{t}\right)=0$, we have

$$
R_{t}^{n-k}+t \sum_{j \neq k, n}\left|a_{j}\right| R_{t}^{j-k}=\left|a_{k}\right|,
$$

which proves the right side of (4).

We prove the left side by contradiction. Suppose, on the contrary, that

$$
R_{t}<\left(1-\frac{\varepsilon}{n}\right)\left|a_{k}\right|^{1 /(n-k)} \text {. }
$$

Then

$$
\begin{aligned}
R_{t}^{n-k}+t \sum_{j \neq k, n}\left|a_{j}\right| R_{t}^{j-k} & <\left(1-\frac{\varepsilon}{n}\right)^{n-k}\left|a_{k}\right|+A_{k} R_{t}^{n-k-1} \\
\leqslant\left(1-\frac{\varepsilon}{n}\right)^{n-k}\left|a_{k}\right|+A_{k}\left(1-\frac{\varepsilon}{n}\right)^{n-k-1}\left|a_{k}\right|^{1-\frac{1}{n-k}} & =\left(1-\frac{\varepsilon}{n}\right)^{n-k}\left|a_{k}\right|+\frac{A_{k}}{\left|a_{k}\right|^{1 /(n-k)}}\left(1-\frac{\varepsilon}{n}\right)^{n-k-1}\left|a_{k}\right| .
\end{aligned}
$$

It follows from (2) that

$$
\frac{A_{k}}{\left|a_{k}\right|^{1 /(n-k)}} \leqslant \frac{\varepsilon}{n}
$$

therefore,

$$
R_{t}^{n-k}+t \sum_{j \neq k, n}\left|a_{j}\right| R_{t}^{j-k}<\left(1-\frac{\varepsilon}{n}\right)^{n-k}\left|a_{k}\right|+\frac{\varepsilon}{n}\left(1-\frac{\varepsilon}{n}\right)^{n-k-1}\left|a_{k}\right|
$$




$$
=\left(1-\frac{\varepsilon}{n}\right)^{n-k-1}\left|a_{k}\right| \leqslant\left|a_{k}\right|,
$$

which contradicts with (5). Thus (4) is proved.

It follows from (3), (4) and the Lemma 1 that $k$ roots of $g_{t}$ lie inside the circle $\{z \in \mathbb{C}:|z|=1\}$ and the other $n-k$ - outside the circle $\{z \in \mathbb{C}:|z|=$ $\left.(1-\varepsilon / n)\left|a_{k}\right|^{1 /(n-k)}\right\}$ for all $t \in[0,1]$.

Let $z_{0}$ be a root of $g_{t}$ from the second group, i.e.,

$$
\left|z_{0}\right|>\left(1-\frac{\varepsilon}{n}\right)\left|a_{k}\right|^{1 /(n-k)} .
$$

We have

$$
\left|z_{0}^{n}+a_{k} z_{0}^{k}\right|=t \cdot\left|\sum_{j \neq k, n} a_{j} z_{0}^{j}\right| \leqslant A_{k} z_{0}^{n-1}
$$

which leads to

$$
\prod_{j=1}^{n-k}\left|z_{0}-w_{j}\right| \leqslant A_{k}\left|z_{0}\right|^{n-k-1} .
$$

This implies that there exists an index $l$ such that

$$
\left|z_{0}-w_{l}\right| \leqslant\left(\frac{A_{k}}{\left|z_{0}\right|}\right)^{1 /(n-k)}\left|z_{0}\right|
$$

Combining this with (2) and (6) we obtain

$$
\begin{aligned}
\left|z_{0}-w_{l}\right|<\left(\frac{A_{k}}{(1-\varepsilon / n)\left|a_{k}\right|^{1 /(n-k)}}\right)^{1 /(n-k)}\left|z_{0}\right| & \\
& \leqslant \frac{\varepsilon}{n+\varepsilon}\left|z_{0}\right| \leqslant \frac{\varepsilon}{n+\varepsilon}\left|w_{l}\right|+\frac{\varepsilon}{n+\varepsilon}\left|z_{0}-w_{l}\right|,
\end{aligned}
$$

which produces

$$
\left|z_{0}-w_{l}\right|<\frac{\varepsilon}{n}\left|w_{l}\right|=\frac{\varepsilon}{n}\left|a_{k}\right|^{1 /(n-k)} .
$$

It means that all roots of $g_{t}$ from the second group belong to $\cup_{m=1}^{n-k} B_{m}$, where $B_{m}=\left\{z \in \mathbb{C}:\left|z-w_{m}\right|<\varepsilon\left|w_{m}\right| / n\right\}$. Since $\varepsilon / n<\sin [\pi /(n-k)]$, all $B_{1}, \ldots, B_{n-k}$ are disjoint. Therefore $g_{t}$ does not vanish on the boundary of $B_{m}$ for all $t \in$ $[0,1], m=1, \ldots, n-k$. To conclude the proof, it remains to show that every $B_{m}$ contains exactly one root of $g_{t}$. Obviously, this is true for $t=0$. Therefore, by Lemma 2 this is also true for all $t \in[0,1]$.

Lemma 4. Let $\left\{\eta_{j}\right\}_{j=0}^{\infty}$ be non-negative i.i.d. random variables. Put

$$
S_{n}=\sum_{j} \eta_{j}, \quad M_{n}=\max \left\{\eta_{j}\right\}_{j=0}^{n} .
$$

(a) The distribution of $\eta_{0}$ has a slowly varying tail if and only if

$$
\frac{M_{n}}{S_{n}} \stackrel{P}{\rightarrow} 1, \quad n \rightarrow \infty .
$$

(b) The distributin of $\eta_{0}$ has an infinite mean if an only if

$$
\frac{S_{n}-M_{n}}{n} \stackrel{\text { a.s. }}{\rightarrow} \infty, \quad n \rightarrow \infty .
$$

Proof. For (a) see [1, for (b) see [5, Theorem 2.1]. 
Lemma 5. Suppose $a_{0}, a_{1}, \ldots, a_{n} \geqslant 0$ and $\varepsilon>0$. If for some $k=1, \ldots, n-1$

$$
\prod_{j \neq k}\left(1+a_{j}\right)^{2 n^{2}} \leqslant 1+a_{k}
$$

and

$$
a_{k} \geqslant 2(1-\varepsilon)^{-4 n^{2} /(4 n-1)} \varepsilon^{-4 n^{3} /(4 n-1)}(n+\varepsilon)^{4 n^{3} /(4 n-1)},
$$

then

$$
\sum_{j \neq k} a_{j}+1 \leqslant\left(1-\frac{\varepsilon}{n}\right)\left(\frac{\varepsilon}{n+\varepsilon}\right)^{n-k} a_{k}^{1 /(n-k)} .
$$

Proof. Since $1+\sum_{j \neq k} a_{j} \leqslant \prod_{j \neq k}\left(1+a_{j}\right)$, it suffices to show that

$$
\left(2 a_{k}\right)^{1 /(2 n)^{2}} \leqslant(1-\varepsilon)\left(\frac{\varepsilon}{n+\varepsilon}\right)^{n} a_{k}^{1 / n}
$$

which is equivalent to (7).

\section{Proof of theorems}

Proof of Theorem 1. By Lemma 4 (a), for any $\delta>0$ we have $\mathbf{P}\left\{A_{n}\right\} \rightarrow 1, n \rightarrow \infty$, where

$$
A_{n}=\left\{\left|\xi_{\tau}\right|>e^{\delta} \sum_{j \neq \tau}\left|\xi_{j}\right|\right\}
$$

Consider the associated polynomial

$$
\tilde{G}(z)=\sum_{j \neq \tau}\left|\xi_{j}\right| z^{j}-\left|\xi_{\tau}\right| z^{\tau} .
$$

Suppose $A_{n}$ occurs. If $1 \leqslant t \leqslant e^{\delta / n}$, then

$$
\left|\xi_{\tau} t^{\tau}\right|>e^{\delta} \sum_{j \neq \tau}\left|\xi_{j}\right| \geqslant t^{n} \sum_{j \neq \tau}\left|\xi_{j}\right| \geqslant\left|\sum_{j \neq \tau} t^{j} \xi_{j}\right| .
$$

If $e^{-\delta / n} \leqslant t \leqslant 1$, then

$$
\left|\xi_{\tau} \tau^{\tau}\right| \geqslant e^{-\delta}\left|\xi_{\tau}\right|>\sum_{j \neq \tau}\left|\xi_{j}\right| \geqslant\left|\sum_{j \neq \tau} t^{j} \xi_{j}\right|
$$

Therefore $\tilde{G}$ does not have real roots in the interval $\left[e^{-\delta / n}, e^{\delta / n}\right]$. Further, $\tilde{G}(0)>$ $0, \tilde{G}(\infty)>0$, and $\tilde{G}(1)<0$. By Descarte's rule of signs $\tilde{G}$ has at most 2 positive roots. Thus $\tilde{G}$ has exactly 2 positive roots $r$ and $R$ such that

$$
0<r<e^{-\delta / n}<e^{\delta / n}<R .
$$

By Lemma 1, $G$ has exactly $\tau$ roots inside the circle $\left\{z \in \mathbb{C}:|z|=e^{-\delta / n}\right\}$ and $n-\tau$ roots outside the circle $\left\{z \in \mathbb{C}:|z|=e^{\delta / n}\right\}$. Therefore, $A_{n}$ implies that $R_{n}\left(e^{-\delta / n}, e^{\delta / n}\right)=0$ which concludes the proof.

Proof of Theorem Q . Consider the events

$$
A_{n}=\left\{\prod_{j \neq \tau}\left(1+\frac{\left|\xi_{j}\right|}{\left|\xi_{n}\right|}\right)^{2 n^{2}} \leqslant 1+\frac{\left|\xi_{\tau}\right|}{\left|\xi_{n}\right|}\right\}
$$


and

$$
B_{n}=\left\{\frac{\left|\xi_{\tau}\right|}{\left|\xi_{n}\right|} \geqslant 2(1-\varepsilon)^{-4 n^{2} /(4 n-1)} \varepsilon^{-4 n^{3} /(4 n-1)}(n+\varepsilon)^{4 n^{3} /(4 n-1)}\right\} .
$$

Since the distribution of $\log \left(1+\log \left(1+\left|\xi_{0}\right|\right)\right)$ has a slowly varying tail, by Lemma प(a),

$$
\mathbf{P}\left\{4 \cdot \sum_{j \neq \tau} \log \left(1+\log \left(1+\left|\xi_{j}\right|\right)\right) \leqslant \log \left(1+\log \left(1+\left|\xi_{\tau}\right|\right)\right)\right\} \rightarrow 1, \quad n \rightarrow \infty,
$$

which implies

$$
\mathbf{P}\left\{\left(\sum_{j \neq \tau} \log \left(1+\left|\xi_{j}\right|\right)\right)^{4} \leqslant \log \left(1+\left|\xi_{\tau}\right|\right)\right\} \rightarrow 1, \quad n \rightarrow \infty .
$$

Since $\mathbf{E} \log \left(1+\left|\xi_{0}\right|\right)=\infty$, by Lemma 4 (b) with probability one

$$
\frac{1}{n} \sum_{j \neq \tau} \log \left(1+\left|\xi_{j}\right|\right) \rightarrow \infty, \quad n \rightarrow \infty,
$$

which together with (8) produces

$$
\mathbf{P}\left\{n^{3} \cdot \sum_{j \neq \tau} \log \left(1+\left|\xi_{j}\right|\right) \leqslant \log \left(1+\left|\xi_{\tau}\right|\right)\right\} \rightarrow 1, \quad n \rightarrow \infty,
$$

and

$$
\mathbf{P}\left\{\log \left(1+\left|\xi_{\tau}\right|\right) \geqslant n^{4}\right\} \rightarrow 1, \quad n \rightarrow \infty .
$$

Since for any $\delta>0$ there exists $T>0$ such that $\mathbf{P}\left\{T^{-1}<\left|\xi_{n}\right|<T\right\}>1-\delta$, the last two inequalities imply

$$
\mathbf{P}\left\{A_{n}\right\}, \mathbf{P}\left\{B_{n}\right\} \rightarrow 1, \quad n \rightarrow \infty .
$$

By Lemma 5 the event $A_{n} \cap B_{n}$ implies that the polynomial $G_{n}(z) /\left|\xi_{n}\right|$ satisfies the conditions of Lemma 3. Thus we have proved the theorem for the roots of $G_{n}$ lying outside the unit circumference. To treat the rest of the roots consider the associated polynomial

$$
G_{n}^{*}(z)=z^{n} G(1 / z)=\sum_{j} \xi_{j} z^{n-j}
$$

and note that $z_{0}$ is a root of $G_{n}$ if and only if $z^{-1}$ is a root of $G_{n}^{*}(z)$.

\section{REFERENCES}

[1] D. Darling, The Influence of the Maximum Term in the Addition of Independent Random Variables, Trans. Amer. Math. Soc., 73 (1952), pp. 95-107.

[2] J. M. Hammersley, The zeroes of a random polynomial, in Proceedings of the Third Berkeley Symposium on Mathematical Statistics and Probability. Vol. II, University California Press, Berkeley, Los Angeles, 1956, pp. 89-111.

[3] I. Ibragimov and O. Zeitouni, On roots of random polynomials, Trans. Amer. Math. Soc., 349 (1997), pp. 2427-2441.

[4] I. Ibragimov and D. Zaporozhets, On distribution of zeros of random polynomials in complex plane, arXiv:1102.3517 1,2011 (to appear in Prokhorov Festschrift)

[5] H. Kesten and R. Maller, The Effect of Trimming on the Strong Law of Large Numbers, Proc. London Math. Soc., 71 (1995), pp. 441-480. 
[6] A. M. Ostrowski, Solution of equations in Euclidean and Banach spaces, New York: Academic Press, 1973.

[7] Q. I. Rahman and G. Schmeisser, Analytic theory of polynomials, New York: Oxford University Press, 2002.

[8] L. Shepp And R.J. VAnderbei, The complex zeros of random polynomials, Trans. Amer. Math. Soc., 347 (1995), pp. 4365-4383.

[9] D. I. Shparo And M. G. Shur, On the distribution of roots of random polynomials, Vestnik Moskov. Univ. Ser. I Mat. Meh., 3 (1962), pp. 40-43. (in Russian)

[10] D. N. Zaporozhets, An example of a random polynomial with unusual zeros behavior, Teor. Ver. Prim., 50 (2005), pp. 549-555. (English translation: Theory Probab. Appl. ) 